\section{Color blindness}

To the Editor: As Bang Wong notes in his recent column on proper color coding in figures, just picking suitable colors is not always sufficient ${ }^{1}$. In particular, one important, but often neglected, issue is color blindness because it affects a substantial portion of the human population (additional information is available at MedlinePlus: http://www.nlm. nih.gov/medlineplus/colorblind ness.html). One well-known example of frequent color vision defects
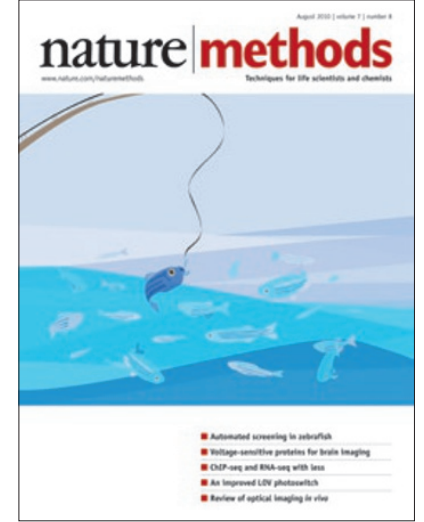
is red-green deficiency, which occurs, in case of Northern European ancestry, in about 8 percent of males and 0.5 percent of females ${ }^{2}$. Therefore, it is advisable to select figure colors accordingly, and journals might introduce color guidelines to increase the authors' awareness of this accessibility issue. For instance, software such as Adobe Photoshop offers color blindness proofing filters that simulate what an image will look like to individuals with color blindness. Authors can then adjust the image to make it universally accessible (see Adobe Photoshop CS5 accessibility overview at http://www.adobe.com/accessibility/products/photoshop/ overview.html).

\section{COMPETING FINANCIAL INTERESTS}

The author declares no competing financial interests.

\section{Mario Albrecht}

Max Planck Institute for Informatics, Campus E1.4, Saarbrücken, Germany. e-mail: mario.albrecht@mpi-inf.mpg.de

1. Wong, B. Nat. Methods 7, 573 (2010).

2. Deeb, S.S. Clin. Genet. 67, 369-377 (2005).

Nature Methods replies: We agree with Albrecht that color blindness in readers is an important but often overlooked consideration when authors choose figure colors ${ }^{1}$. In 2007 Nature Publishing Group began recommending that authors choose color combinations other than green and red when they prepare their manuscripts for submission. Before accepting any manuscript, we again ask authors to recolor red-and-green graphs, schematics and models in which the colors are arbitrary. Unfortunately, as can be seen in the pages of this issue and issues of other Nature Publishing Group journals, there are still instances of red and green being used in a manner inconsistent with these guidelines. We hope to highlight this issue in a future Points of View column that provides more detailed guidelines on choosing colors that are accessible to our readers with color blindness. In the meantime, we encourage authors to visit http://www.vischeck.com/ for more information and to download their free Photoshop and ImageJ plug-ins for recoloring images.

1. Albrecht, M. Nat. Methods 7, 775 (2010).

\section{Rapid readout detector captures protein time-resolved WAXS}

To the Editor: Time-resolved wide-angle X-ray scattering (WAXS) is emerging as a powerful approach for visualizing global conformational changes in proteins in real time. WAXS-based studies to date include following the light-triggered reactions of hemoglobin ${ }^{1}$ and myoglobin ${ }^{2,3}$ in complex with carbon monoxide, and structural characterization of the photocycles of bacteriorhodopsin and proteorhodopsin ${ }^{4}$. Compared to time-resolved Laue diffraction and trapped intermediate studies ${ }^{5}$, time-resolved WAXS is a solution-based method and therefore permits visualization
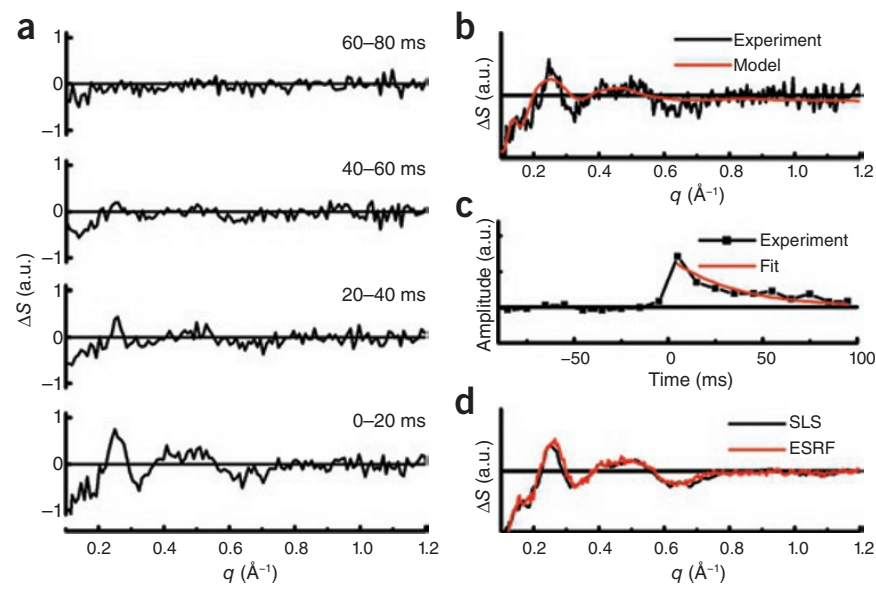

Figure 1 | Time-resolved WAXS difference data recorded from detergentsolubilized samples of proteorhodopsin. (a) Experimental difference data for the indicated time windows after phototriggering. $\Delta S$ is the change in X-ray scattering intensity, and $q=4 \pi \sin (\theta) / \lambda$, where $\theta$ is half the angle through which $X$-rays are deflected and $\lambda$ is the $X$-ray wavelength. (b) Singular value decomposition of the experimental difference data: the principal component (difference WAXS basis spectrum; experiment) and the results of structural refinement against this basis spectrum (model; see ref. 4). (c) Time evolution of the principal component shown in $\mathbf{b}$ as recovered by singular value decomposition (experiment) and the fit to a monoexponential decay (fit). (d) The difference WAXS basis spectrum recorded from proteorhodopsin ${ }^{4}$ using short polychromatic X-ray pulses at the European Synchrotron Radiation Facility (ESRF) overlaid upon that recorded using monochromatic radiation at the Swiss Light Source (SLS) after convolution with the polychromatic $\mathrm{X}$-ray spectrum. 